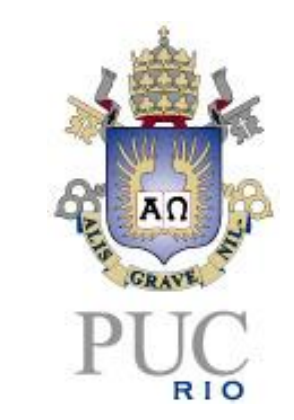

Cleber Fernandes Taboza

\title{
A Relação Intertemporal entre o Value at Risk e os Retornos Esperados no Mercado Brasileiro
}

Dissertação de Mestrado

Dissertação apresentada como requisito parcial para obtenção do título de Mestre pelo Programa de Pós-Graduação em Administração de Empresas do Departamento de Administração da PUC-Rio.

Orientador: Prof. Antonio Carlos Figueiredo Pinto

Rio de Janeiro

Março de 2013 


\title{
A Relação Intertemporal entre o Value at Risk e os Retornos Esperados no Mercado Brasileiro
}

\begin{abstract}
Dissertação apresentada como requisito parcial para obtenção do título de Mestre pelo Programa de PósGraduação em Administração de Empresas do Departamento de Administração da PUC-Rio. Aprovada pela Comissão Examinadora abaixo assinada.
\end{abstract}

Prof. Antonio Carlos Figueiredo Pinto

Orientador

Departamento de Administração, PUC-Rio

Prof. Marcelo Cabus Klotzle

Departamento de Administração, PUC-Rio

Prof. Claudio Henrique da Silveira Barbedo

Banco Central do Brasil e IBMEC-RJ

Prof ${ }^{a}$. Mônica Herz

Vice-Decana de Pós-Graduação do CCS - PUC-Rio 
Todos os direitos reservados. É proibida a reprodução total ou parcial do trabalho sem autorização da universidade, do autor e do orientador.

Cleber Fernandes Taboza

Graduou-se em Ciências Contábeis (2000) e em Direito (2008), ambos pela UFRJ. É funcionário do Banco Central do Brasil, exercendo o cargo de analista no Departamento de Monitoramento do Sistema Financeiro.

Ficha Catalográfica

Taboza, Cleber Fernandes

A relação intertemporal entre o Value at Risk e os retornos esperados no mercado brasileiro / Cleber Fernandes Taboza; orientador: Antonio Carlos Figueiredo Pinto. - 2013.

$51 \mathrm{f} . ; 30 \mathrm{~cm}$

Dissertação (mestrado)-Pontifícia Universidade Católica do Rio de Janeiro, Departamento de Administração, 2013.

Inclui bibliografia

1. Administração - Teses. 2. Value at Risk. 3. Retornos esperados. 4. ICAPM. 5. Risco-retorno. I. Pinto, Antonio Carlos Figueiredo. II. Pontifícia Universidade Católica do Rio de Janeiro. Departamento de Administração. III. Título. 
À minha avó Eliza (in memoriam), pela dedicação e amor com que me introduziu no mundo das letras e dos números.

À Michele, pelo amor e parceria nesta já longa jornada. 


\section{Agradecimentos}

À minha mãe e demais familiares, por terem compreendido as poucas visitas e também por terem me pressionado (ainda que de forma velada) para que estivesse presente nos principais momentos. Isto me obrigou ao saudável desligamento tão necessário em projetos de longa duração.

Ao Professor Figueiredo, por me mostrar a direção certa nos momentos mais críticos.

Aos Professores Marcelo Cabus, Luiz Felipe e André Carvalhal, pelo ensino de excelência.

À PUC-Rio, pelos auxílios concedidos, sem os quais este trabalho não poderia ter sido realizado.

Aos colegas Cláudio Barbedo e Wagner Gaglianone, pelas preciosas sugestões.

Ao Banco Central do Brasil, pela oportunidade de me aprimorar intelectualmente. Sem o suporte material do Bacen esta conquista não seria possível ou seria muito mais penosa.

Aos amigos do Desig/Bacen, em especial o Alexandre Moraes e o Edson Broxado, por terem acreditado que posso agregar à equipe e nela terem me recebido após a conclusão do mestrado.

Aos amigos do mestrado. Tive o privilégio de fazer parte de um grupo cooperativo, bem humorado e dedicado. Isto enriqueceu bastante essa fase da minha vida. Agradeço em especial aos amigos Marcelo, Matheus, Vinay e Vinicius.

À Teresa e ao Fábio, pelo carinho e dedicação com que tratam os pósgraduandos toda vez que precisamos do suporte da secretaria acadêmica. 


\section{Resumo}

Taboza, Cleber Fernandes; Pinto, Antonio Carlos Figueiredo (orientador). A Relação Intertemporal entre o Value at Risk e os Retornos Esperados no Mercado Brasileiro. Rio de Janeiro, 2013. 51p. Dissertação de Mestrado - Departamento de Administração, Pontifícia Universidade Católica do Rio de Janeiro.

Diversos estudos têm procurado uma variável de risco que empiricamente tenha uma relação positiva e significativa com os retornos condicionais de mercado. Na maior parte dos casos a escolha recai sobre novas abordagens envolvendo a variância condicional dos retornos. Neste trabalho substituímos a variância pelo Value at Risk (VaR) para analisar se no mercado brasileiro existe o trade-off entre risco e retorno. O VaR é estimado paramétrica e não parametricamente com base em janelas de dados de um a seis meses. Os resultados mostram que em nosso mercado não há relação positiva e significativa entre o $\mathrm{VaR}$ e os retornos mensais. A causa mais aparente para essa divergência é que o prêmio de risco de mercado é negativo em 114 dos 217 meses que compõem a série temporal da variável dependente, impactando os coeficientes do VaR nas regressões. Quando utilizados retornos com frequência diária, os resultados mostram que em períodos mais recentes há relação positiva e significativa entre esses retornos e o VaR paramétrico.

\section{Palavras-chave}

Value at Risk; retornos esperados; ICAPM; risco-retorno. 


\section{Abstract}

Taboza, Cleber Fernandes; Pinto, Antonio Carlos Figueiredo (Advisor). The Intertemporal Relation between the Value at Risk and the Expected Returns in the Brazilian Market. Rio de Janeiro, 2013. 51p. MSc. Dissertation - Departamento de Administração, Pontifícia Universidade Católica do Rio de Janeiro.

Several studies have searched a risk variable with an empirically positive and significant relation with excess market returns. At the most part of the cases the choices are new approaches of conditional variance of the returns. In this paper we substitute the variance for the Value at Risk (VaR) to analyze whether in the Brazilian market there is relation between risk and returns. The VaR is estimated in parametric and nonparametric ways, considered the precedents intervals of time from one to six months. The results show that in our market there is not a positive and significant relation between $\mathrm{VaR}$ and the monthly market returns. The most obvious cause that supports our results is that the market premium risk is negative on 114 of 217 total monthly observations that form the temporal series of the dependent variable, impacting the VaR coefficients in the regressions. When used daily frequency returns, the results show a positive and significant relation between these results and parametric $\mathrm{VaR}$ in recent periods.

\section{Keywords}

Value at Risk; expected returns; ICAPM; risk-return. 


\section{Sumário}

1 Introdução 11

1.1 Objetivos 13

1.2 Relevância do Estudo 14

$\begin{array}{ll}1.3 \text { Delimitação do Estudo } & 14\end{array}$

2 Revisão da Literatura 16

$\begin{array}{ll}2.1 \text { Risco } & 16\end{array}$

2.2 Intertemporal Capital Asset Pricing Model 19

2.3 Value at Risk e Retornos de Mercado 20

3 Metodologia e Amostra 23

3.1 Descrição da Amostra 23

3.1.1 Augmented Dickey-Fuller (ADF) 26

3.2 Metodologia 28

3.2.1 Retornos de Mercado 28

3.2.2 VaR não Paramétrico 29

3.2.3 VaR Paramétrico 32

4 Análise dos Resultados 35

4.1 Resultados das Regressões para o VaR não Paramétrico 38

4.2 Resultados das Regressões para o VaR Paramétrico 40

4.3 Resultados das Reg. Utilizando Retornos com Frequência Diária 41

5 Conclusão

6 Referências $\quad 46$

$\begin{array}{ll}7 \text { Anexos } & 49\end{array}$ 


\section{Lista de Tabelas}

Tabela 1 - Estatísticas descritivas das séries mensais

Tabela 2 - Resultados para o Augmented Dickey-Fuller T (ADF)

Tabela 3 - Estatísticas descritivas do VaR não paramétrico

Tabela 4 - Estatísticas descritivas do VaR paramétrico

Tabela 5 - Resultados do teste de Chow para os resíduos de regressões utilizando o VaR não paramétrico (antes da inclusão das variáveis dummies)

Tabela 6 - Resultados do teste de Chow para os resíduos de regressões utilizando o $\mathrm{VaR}$ paramétrico (antes da inclusão das variáveis dummies)

Tabela 7 - Resultados das regressões utilizando o VaR não paramétrico

Tabela 8 - Resultados das regressões utilizando o VaR paramétrico

Tabela 9 - Resultados das regressões utilizando retornos com frequência diária e VaR paramétrico 


\section{Lista de Gráficos e Anexos}

Gráfico 1 - Evolução dos retornos do Ibovespa, dos retornos do CDI e do excesso de retorno de mercado

Gráfico 2 - Resíduos da regressão executada utilizando o VaR não paramétrico estimado com três meses de dados

Gráfico 3 - Resíduos da regressão executada utilizando o VaR paramétrico estimado com três meses de dados

Anexo 1 - Resíduos das regressões antes da inclusão das variáveis dummies (usando o VaR não paramétrico estimado em diversos intervalos)

Anexo 2 - Resíduos das regressões antes da inclusão das variáveis dummies (usando o VaR paramétrico estimado em diversos intervalos)

Anexo 3 - Resultados das regressões utilizando retornos com frequência diária e VaR paramétrico (complemento da tabela 9) 